\title{
Information Technology as Social Phenomenon
}

\author{
Daniel Memmi \\ Computer Science Dept. \\ University of Quebec in Montreal \\ Montreal, QC, Canada \\ memmi.daniel@uqam.ca
}

\begin{abstract}
Computer science is of course first of all a technological domain, but it has also become an important social phenomenon as well. Information processing techniques fulfill crucial social functions and give rise to novel forms of social organization. Computer-mediated electronic networks make possible highly distributed, interactive communication patterns corresponding closely to modern social trends. We intend to analyze here the close interplay of social changes and technological advances, which underlies much of the evolution in this domain. We will see with several examples how technology is essentially embedded within global human culture and in constant interaction with social structures. This will enable us to better understand recent social developments online, as well as to suggest promising directions for technological developments.
\end{abstract}

Keywords computer science, information technology, communication processes, sociology, social networks, social media, knowledge management, virtual institutions, technology and society.

\section{Introduction}

Computer science is primarily a technology, i.e. a congruent set of techniques and methods, which is often described in this particular case as the combination of hardware and software. But this technology has also had a huge influence on social phenomena and social organization, and such an influence should be of interest not only for sociologists and economists, but for computer scientists as well. Conversely, technological development is dependent on social trends, and this should also interest researchers and engineers. Clearly, the context and use of a technology are relevant to its developers, and they had better take them into account in their work.

This is not really new: previous waves of technological change have had enormous influence on social and economic structures in their time. The steam engine, railroads, electricity, the telephone, the radio, cars and planes for example have all deeply changed society in many ways. These various techniques have introduced a new mobility and a speed of communication which have transformed social structures. These past waves have been described and discussed at length, but much remains to be said about the current wave of information processing, in spite of recent discussions. 
What is specific about the computer revolution is how closely intertwined it is with communication processes in our society. Most communication exchanges by now take place through computer-operated networks. Computer technology is thus situated at the core of social interactions, in intimate contact with social trends, resulting in tighter and quicker reciprocal influences between technology and society than in previous technological waves.

Terminology is significant here. In English, technical people tend to speak of computer science, but business types often use information processing or information technology (IT), stressing function rather than technique. In French, one calls it informatique ("informatics" or similar words in other European languages) i.e. the science of information, but also technologies de l'information et de la communication ("information and communication technology") to stress the communicative function of information technology.

As a matter of fact, the predominant use of computer technology by now has become communication between human beings, so we will mostly talk here of information technology (IT). The internet has become the backbone of most communication channels: e-mail, instant messaging, social media, as well as phone communications (by voice or text) are all part of this giant network of networks. Increasingly, all forms of human communication at a distance tend to take place on the internet and its numerous applications.

Only mass media such as television, radio and the press have kept to their own (noninteractive) distribution channels for a time (Hertzian broadcasting and paper delivery respectively), but with digitalization, streaming and programming on demand, they are increasingly offered on electronic networks as well. The paper press is rapidly giving way to web sites (not without qualms) and the young tend to access television or radio programs on various portable devices connected to the internet.

Smartphones are the perfect example of this generalized merging of voice and text communication with access to the internet and the web. Their huge success is due precisely to this fusion of hitherto separate capabilities, which used to require different devices. Portability is another factor: internet access is now available practically anywhere anytime on handheld devices.

All this is well known by now. But it is worthwhile to state explicitly the extent and consequences of this global evolution: most human communication (other than face-to-face) is now managed by computers, and computers are used mostly to manage human communication.

Of course, computers are indispensable (and more and more so) to manage accounting, banks, factories and hospitals, do scientific computing, run simulations, pilot airplanes and drive robots. There is in fact more and more computing done within all kinds of common devices, from elevators and cars to cameras and washing machines. But these undoubtedly very useful applications are simply dwarfed by the sheer mass and rapid development of electronic communication usage nowadays.

In this text, we intend to show the social importance of information technology and we will try to analyze in some detail the interplay of social changes and technical evolution that takes place in this domain. Without pretending to be exhaustive, we will describe a few typical examples of the close relations between technology and society. We will see how social trends interact with technological developments, and will then try to clarify the causes and effects involved in this process. Beside the theoretical interest of such a discussion, it should help develop relevant and successful technical applications. 


\section{The example of PageRank}

In order to illustrate the symbiosis of technology and society inherent in the development of information technology, we will first use a famous example: Google's web search algorithm. The web exhibits a very particular structure, which can be ultimately explained by human behavior: well-known sites tend to attract more new links ("preferential attachment"), resulting in a highly skewed connectivity pattern, with many connections attached to few sites (Barabasi \& Reka 1999). This graph structure can be exploited to improve search results.

Google started its remarkable development by launching a new search engine based on a clever algorithm, PageRank (Brin \& Page 1998). Google's search engine quickly captured most of the market for web searches by returning web pages sorted by order of relevance, thus making search results much more useful and satisfying. PageRank rates retrieved pages according to their popularity: the algorithm gives a higher rank to web pages cited by the highest number of pages that are themselves pointed to by other popular pages (and so on recursively).

This ranking corresponds to a mathematical concept, the eigenvector of a matrix: the first eigenvector of the matrix of connections between web pages provides a popularity score for each page. This rating can reasonably be used as a measure of relevance, as it represents a kind of vote of confidence in a given page by other pages (i.e. by the human designers of these pages). The ranking can be readily computed, because computing eigenvectors has long been an important problem with many applications.

The actual PageRank algorithm is in fact more complicated, and its implementation has been refined repeatedly. But the basic idea lies in the application of a social notion to the solution of a technical problem, exploiting the structure of the web to return the most relevant web pages first. The ranking used is indeed a variant of eigenvector centrality, a well-known measure of influence which has been used in bibliometrics and sociology (Wasserman \& Faust 1994). Eigenvector centrality has been employed in particular to give a precise mathematical formulation of an individual's "social capital", i.e. the wealth in social connections that is associated with social influence and social success.

Our main point here is that the designers of PageRank noticed that the structure of the web is a social construction, understood that crucial semantic information could be extracted from this structure, and found a clean and efficient way to do so. They solved an important technical problem by exploiting social information, thus fulfilling an important social need for relevant information. But this is just one example of the interaction of technology and society.

\section{Social context of technology}

By taking a long view, one may observe how much electronic communication fits in with global trends toward a more open and fragmented society, decentralized management of business firms, and the growth of a knowledge economy based on human expertise and constant innovation. Let us now analyze each of these aspects in turn.

\subsection{Social trends}

It is one the basic tenets of sociology that our modern urbanized, industrial and bureaucratic society has evolved from closed communities toward open, more impersonal groupings (Tönnies 
1963; Weber 1956). The German sociologists that first described this phenomenon at the end of the 19th century called it a transition from Gemeinschaft (community) to Gesellschaft (society).

A traditional community (Gemeinschaft) is a mostly closed, densely linked, stable social group where people know each other on a personal basis. Community members live in the same locality (a village for example) and communication can take place face-to-face.

Modern society (Gesellschaft) is usually of larger size, more sparsely linked, geographically scattered, so that its members often do not know each other personally any longer. Group membership is flexible, freer from personal allegiances, and relations tend to be more impersonal, as social status and interactions are determined by functional considerations instead (notably by work position and financial means). Such a society is more and more fragmented and requires explicit rules and communication channels for its regular operations.

Apart from different social and psychological aspects (constraints and security of traditional community vs. freedom and loneliness of modern society), these two society types also have different communication requirements. The increasing size, diversity, flexibility and fragmentation of modern society have called for various communication devices: point-to-point techniques such as postal mail, telegraph, telephone, telex, as well as broadcasting devices like the press, radio and later television...

Changing social structures have probably motivated the development of research in social networks (i.e. the formal structure of social relations). The domain has been mathematically formalized to a large extent and many studies are now available (Wasserman \& Faust 1994; Lazega 1998).

It is in this context that recent electronic communication devices have come into play to ensure communication between the distant, varied and autonomous members of modern industrial (or postindustrial) society, who are constantly rearranging their social links, at work and in their private life (Wellman 1999). The fluidity of modern social life requires either a physical presence in the same location (hence the importance of cities and rapid transit) or rapid, easy to use communication devices (from the telephone to the internet).

The explosive success of social media (such as Facebook or Twitter) must also be seen in this social context. Electronic applications are tools used to manage social relations and information diffusion in a fragmented, dispersed and flexible society, and also to manage modern economic organizations.

\subsection{Management structure}

Recent economic evolutions reinforce the need for efficient, easy-to-use communication techniques. Globalization, outsourcing of production, and flexible work organization methods require increased capacities for communication between economic partners, often at a distance (Castells 1996; Veltz 2000). The growth of international commerce in particular, due to more open markets and to the container revolution in shipping, requires rapid long-distance communication.

The tendency to distribute design and production among networks of firms, often located on distant sites, obviously calls for good co-ordination techniques. Giant firms such as Toyota manage a huge network of various suppliers, spread over several countries, a problem made worse by the exigencies of "just-in-time" delivery methods. And "hollow firms" retain only management and design functions, while systematically outsourcing production and distribution 
(Nike is a good example). This evolution has been both beneficial and disruptive, but it is a massive fact of today's world.

To ensure the coherent management of such dispersed, heterogeneous economic partners, firms make use of the whole panoply of electronic communication techniques: e-mail, interactive web sites, video conference, instant messaging as well as landline and cell phones... Traveling for face-to-face meetings still happens, but is obviously too slow (and too expensive) for daily operations.

In short, modern decentralized management and production methods have been made practically feasible by the speed and easy access of electronic networks, while proving at the same time a powerful incentive for the continuing development of these networks and techniques. Accordingly, governments the world over keep investing huge sums to maintain and upgrade the electronic communication networks that are crucial to economic development.

Another reason for the evolution toward decentralized management methods is the increasing role of technical and scientific knowledge in production processes. One may talk of a knowledge economy based on distributed knowledge-intensive activities, which have their own particular communication requirements.

\subsection{Knowledge economy}

For about 40 years now in developed countries, the stock of investment in intangible assets (teaching, research, R\&D...) has exceeded physical capital (factories, infrastructure). We have gone from an industrial society based on physical capital to a knowledge economy based on human and social capital, i.e. on expertise and expert interactions (Kendrick 1994; Foray 2004).

Knowledge-intensive activities (notably information technology, aerospace, pharmaceutical companies...) are now the most important, wealthiest, dynamic and influential sector of the economy, and traditional industry (automobiles for example) can only survive by incorporating more and more knowledge-intensive processes and components. This "knowledge sector" thrives on the rapid churning and exchange of ideas, methods and qualified staff, again requiring constant and easy access to information and expertise. The knowledge economy typically exhibits a constant flux of high-level information between ever-changing partners.

This knowledge economy is operated by a distinctive class of highly qualified "knowledge workers" (Drucker 1992). Engineers, researchers, technicians share a common technical culture and take pride in their expertise and initiative. They do not conform well to hierarchical discipline, and tend to rely instead on horizontal networks of peers, often cutting across the formal boundaries of firms (Bagla 2003). As these networks are crucial for the acquisition and use of knowledge, management methods have had to adapt to a more flexible social structure.

Because innovation and change have become a major source of growth, strength and resilience in international markets, knowledge and expertise are all the more important (Guellec 1999). In fact, innovation is often necessary for economic survival in the first place. Innovation feeds upon easy access to diversified information, and takes place within flexible social networks spreading across clusters of firms and research institutions, and informal networks of expert professionals. Moreover, suppliers and customers may now contribute to changes as well as designers and producers (Von Hippel 2005).

Here too, firms and individuals make use of the whole gamut of communication techniques, from e-mail to cell phones. Access to relevant information on the web or in databases is also crucial. Yet face-to-face meetings (e.g. in trade fairs or informal meetings) remain probably more 
important than in daily management, because the main motivation here is a slower exchange and buildup of knowledge, and the role of informal professional networks is essential for this purpose.

The evolution toward a highly innovative knowledge economy thus requires a constant flow of knowledge and expertise between various distributed actors, fostering the increased use and development of communication techniques.

\subsection{Cultural consequences}

To sum up, our present society and economy are organized more and more in highly networked (but variable) structures, between individuals, between individuals and organizations, between firms and within firms, distributed among different locations and countries (Castells 1996). Information and knowledge flow through these networks, and the social and productive structure could not function and evolve without this constant stream of information. Hence the crucial importance of modern communication techniques, which have enabled us so far to deal with the management of these complex and flexible social structures.

Such a remarkable social and economic evolution cannot but have important cultural consequences. Generalized and flexible networking gives rise to an almost addictive craving for fleeting, superficial contacts, as well as to feelings of depersonalization and social fragmentation. Social media in particular are both a cause of, and an answer to such anxious feelings. The fact that more and more social interaction is now computer-mediated and takes place online reinforces this impersonal trend even more.

One may also wonder about the increasing complexity of social, technical and economic organizations, and about our capacity to deal with such complexity. At what point does the increased cost of managing complexity exceed the economic benefits of a more complex structure? At what point do we simply become unable to cope with increasing complexity, for lack of appropriate methods or simply because we may fail to grasp what is going on? This may well be one the causes of recent economic crises.

At the same time, another answer to impersonal contacts and increased complexity is a growing tendency to exchange mostly standardized, so-called "objective" information. Standardized information reduces the costs of human interactions, and is best exchanged within a clear social structure. In this way new forms of social organization have begun to emerge online, feeding on previous long-term trends.

\section{Virtual institutions}

With the advent of the internet, "virtual" forms of social life began to take place online. They were seen at first on the model of traditional communities, though possibly with peculiar characteristics (Kollock \& Smith 1999; Rheingold 2000; Memmi 2006). But more complex, goaloriented virtual structures soon appeared (Camarinha-Matos et al. 2004).

In the past decade or so, a new phenomenon has emerged: the gradual buildup of social institutions online (Orman 2010; Memmi 2014). Electronic communication makes it possible to manage elaborate social organizations that operate mainly or exclusively on the internet. These virtual institutions exhibit the main features of classical institutions: values, norms, a social structure, rules and procedures as well as arbitration mechanisms to deal with conflicts. As social 
institutions constitute the building blocks of any society and are central to sociological discourse (Mintzberg 1979; Scott 2001; Miller 2012), the recent developments online are certainly worthy of interest.

Virtual institutions also present original qualities: they are usually collaborative and voluntary, requiring neither buildings nor wages (apart from a small core of administrators) and are thus cheap to operate. Yet this distributed and collaborative model can produce high-quality results at low cost. The model is also flexible, able to react and adapt quickly if necessary. These are very interesting qualities indeed, and we can expect the model to develop and spread even more.

Wikipedia would be a good example, among a few others (Wikipedia 2013). This online encyclopedia is continuously written up and edited collaboratively by numerous anonymous, unpaid volunteers. Contributions are posted on a wiki for all to see and revise if need be. In this way a huge number of very decent articles have been produced within a decade (more than 4 million entries in English, around 1 million entries each in the main other European languages).

To ensure the objectivity and quality of information, the Wikipedia community has gradually elaborated explicit, detailed rules and norms about writing style and conflict resolution. A flexible but fairly stable social structure makes sure that rules are followed and can arbitrate conflicts if necessary. In other words, Wikipedia has become a fully-fledged social institution while operating mostly online.

One could cite other virtual institutions. For example free and open source software projects are developed by the voluntary online collaboration of numerous participants, whose contributions are integrated by a small core of project managers (Ghosh et al. 2002; Feller et al. 2005). In this way free high-quality software has been produced, such as the software managing internet routers. The $\mathrm{W} 3 \mathrm{C}$ consortium supervising the development of the internet also operates mostly online, thanks to the collaboration of technical organizations rather than individual members (W3C 2013).

The general model common to these various online institutions is worth describing in greater detail, both from a sociological perspective and from a technical viewpoint. We could elaborate some more on the novelty and peculiar qualities of this model, as well as the technical challenges it presents. But our main point here is to illustrate the interplay of social trends with technical changes.

If we try to understand in greater detail how virtual institutions came about, we discern a complex web of relations between social and technical factors. First, we may observe that virtual institutions are but a recent incarnation of long-term social trends. The transition from closed communities to a more open and fragmented society has been going on for more than a century, and the growing importance of international trade has fostered decentralized patterns of management. Distributed virtual institutions with numerous, variable contributors fit quite well with this general social evolution: volunteers collaborate on a specific project without forming a community otherwise.

The voluntary and collaborative aspects of virtual organizations (most contributors working for free), although amazing at first sight within a capitalist system, is much more common in society than economic orthodoxy would lead us to believe. Ethnology has described ritualized exchanges of gifts in traditional societies, such as potlatch in North-West America or kula in the Southern Pacific (Mauss 1924; Malinowski 1922). And in our society, voluntary non-profit activity is actually intense and durable, allowing innumerable associations to contribute to all aspects of social life (from neighborhood associations to multinational NGOs). 
Yet virtual institutions have only been made practically possible by recent technical advances, namely cheap, quick and easy-to-use electronic communication. Before the internet, it was much more difficult (and much slower) to co-ordinate the efforts of numerous contributors dispersed over a wide area. Specific applications such as wiki software have also been crucial to manage interactions without too much hassle.

This combination of social trends and technical tools has thus given rise to a new form of social organization online, with novel features that make it a very attractive social model. Virtual institutions have proven to be cheap, reactive, flexible and efficient. But the original features of virtual institutions are an immediate consequence of the communication tools available nowadays.

As they gain momentum and influence, virtual institutions will probably motivate in turn the development of the technical tools they need for their operation: network infrastructure and communication software will have to be updated to accompany these new social usages.

Novel social organizations emerging online are one more proof of the close imbrication of social tendencies with technical possibilities. In a kind of spiral feeding upon itself, technical developments make possible social experiments, which in turn motivate further technical developments, and so on.

\section{Relationship between technology and society}

The interaction of technology and society poses fundamental questions about their precise relationship: which comes first, social change or technical change? Which influences the other? What is the exact chain of causes and effects? We have not yet taken here a clear position on these questions, and it is time to discuss them explicitly. There are at least three possible basic positions on this issue:

- intrinsic technological change

According to this view, technological change happens more or less in isolation, following a kind of inner logic. The development of a technical domain poses internal problems and suggests opportunities, regardless of the social or economic environment. This is often the spontaneous view of technical people, who are understandably more sensitive to events in their field than in the world at large. But historians of science, more aware of wider social influences on technological evolution, tend to consider this view as simplistic. It may also be that intrinsic technological change applies better to periods of continuing progress within the same paradigm, than to sudden shifts in paradigm (Kuhn 1970).

- social trends cause technical changes

In this view, cultural influences determine every human endeavor, and technology is always embedded in a wider culture (this tends to be the view of ethnology). This would explain why some technological changes that seem obvious to us in hindsight do not take place in a particular society, or take an incredibly long time to be fully adopted: technical change is unlikely to happen before the necessary cultural evolution has run its course. Yet this seems more likely to be the case in conservative traditional communities than in modern industrial society, in which systematic research and development has become a norm in itself, and tends to follow its own course. 
- reciprocal influences of technology and society

Many historians of science and technology take the position that there is in fact a regular interplay of technology and society. The sociology of science shows the numerous interactions between social demand and scientific research (Latour 1987; McGinn 1991). According to this more complex view, it would be simplistic to try to isolate first causes in the course of technological development. Technical changes are indeed motivated by socio-economic needs and made possible by cultural attitudes and beliefs. But technical changes accelerate in turn the social trends that might have enabled them in the first place, in a deepening spiral of causes and effects... This is the position we have implicitly taken so far in this text, and we now claim it explicitly.

There are many cases of such interactions of technology and society. For example when motor cars were first developed, they were in fact less efficient and reliable than traditional horse-drawn transportation. The motivation for car development was mostly technical at first, and also part of a wider movement toward mechanized transportation on land, sea and in the air. But little by little cars became cheaper and more efficient, which made it possible for them to replace any other form of urban transportation, if so desired.

Yet cars required adequate infrastructures and support systems (notably good roads and service stations). Decisions were then often made to adapt towns and cities to car transit and to make the automobile the primary mode of transportation. It should be noted that these were political decisions, not technical ones (there were other technical possibilities, such as mass transit systems). But this technical change reshaped cities the world over to a large extent, influencing population patterns, urban geography, economic development. The modern city built around car transit developed its own culture, in which mobility and social fragmentation would then require even more transit infrastructures.

We can see in this familiar example some of the complex interactions between culture, society, economy and technology. Influences play back and forth between these various components, and it seems very difficult to find a primary or fundamental cause in a long chain of events involving many social actors and cultural factors. It is also rather simplistic to speak of technology and society as if they were only two actors in this rather complicated scene.

Going back to information technology, we would conclude that fundamental social trends toward more and more fragmented, impersonal and flexible social structures, and later toward decentralized and distributed management methods, have elicited and fostered the current explosion in electronic communication techniques. But such social and cultural changes were themselves concomitant with the first industrial revolution and the first wave of urbanization, before going on to influence today's society.

Recent advances in communication technology now make possible in turn further advances on the road to fragmentation, flexibility and distributed management, which will then probably demand better communication techniques. The process will only stop when we reach a technical impasse (bandwidth limits for instance) or a social bottleneck (incapacity to deal with the social disruptions involved), but it is likely to take a long time to play out till the next wave of technical changes.

In short, the relationship between technology and society is much more complex than would appear at first sight, and a detailed analysis is necessary to better understand the factors involved and their interactions in a given case. 


\section{Relevance for information technology}

Given the social embedding of information technology, the applications most likely to succeed will fulfill specific social information needs. We have seen several examples, ranging from Google's search engine to social media such as Facebook, which have been extraordinary successful because they are in agreement with the expectations and needs of modern society. Apple's and Samsung's smartphones, merging seamlessly cell phone technology with internet access, also come to mind.

Although the underlying infrastructure and the software running the networks can be quite complex, user-oriented applications may be fairly simple at first. Social media like Facebook were not technically very sophisticated, but quickly proved to be a hugely popular communication tool to help manage the social life of young people deprived of traditional local venues for socializing (the village square does not exist any more, and you need a car to go to the shopping mall). Social relevance has been more important here than technical brilliance.

Going beyond today's successes, the "killer apps" of tomorrow will probably help manage some of the pending problems we have outlined above. As social organizations online become more and more complex and turn into fully-fledged virtual institutions, new needs arise for coordination of distributed actions and conflict resolution between diverse participants. In other words, age-old political issues (consensus-building, decision-making, arbitration procedures) must now be translated into technical problems to be solved online. The problems are in a way perfectly traditional, but they require novel solutions.

We are convinced that the increasing complexity of social life online calls for new software tools to better manage massive collaboration patterns, goal-oriented interactions, structured discussions, conflict resolution... The Wikipedia community shows how to begin to tackle such problems, but in this case solutions have obviously been elaborated piecemeal in the course of years, and a systematic review of the problems might be a good idea. The wiki technique with which most of the interactions take place has proven serviceable, but is still very simple compared to the range of problems involved: a wiki is basically an interactive blackboard with a memory of changes, and little more.

Examining with a fresh eye the requirements of virtual organizations should suggest new ways to help manage social life online. The main problem is probably to formalize communication patterns to some extent (as in any community), but without incurring too much rigidity, which would discourage participants and prevent the flexibility of purposes and processes that is one the typical benefits of virtual organizations.

This might be an interdisciplinary research program, including social scientists together with software engineers. A systematic analysis of the fundamental needs of virtual organizations would show the way for appropriate applications to better manage social life online. Tools to structure and focus discussions, reach consensus, make decisions, arbitrate conflicts, should improve the efficiency and convenience of virtual organizations. Work in this direction has hardly started (except for some voting software) and we think much could be gained by tackling explicitly social co-ordination problems online. 


\section{Conclusion}

We have analyzed the interactions between recent developments in information technology and long-term social changes. We have shown how the amazing expansion of electronic communications techniques based on the internet clearly parallels a global social evolution toward dispersed, flexible and interactive social networks. Information technology fulfills the communication needs of today's society and economy, while at the same time accelerating social change even more.

We have seen in this typical domain how technology is fundamentally embedded within a wider culture. Technology does not develop in a vacuum, but is highly sensitive to social trends and needs, and influences in turn the very society that uses it. Technology and society influence each other in a kind of continuous spiral, and it is probably futile to try to determine which comes first, which is cause or effect at any given time.

Last but not least, such an analysis strongly suggests that software applications most likely to succeed will be those answering the communication needs of changing social structures. A clear and conscious view of social evolution would help develop appropriate and successful technical applications. Awareness of the sociological phenomena involved should prove rewarding for computer scientists in the course of their technical work.

\section{References}

Bagla L. (2003) Sociologie des Organisations, La Découverte, Paris.

Barabasi A.L. \& Reka A. (1999) Emergence of scaling in random networks, Science 286: 509512.

Brin S. \& Page L. (1998) The anatomy of a large-scale hypertextual Web search engine, Computer Science Dept., Stanford University, Calif.

Camarinha-Matos L., Afsarmanesh H. \& Ollus M. (eds) (2004) Virtual Organizations: Systems and Practices, Springer, New York.

Castells M. (1996) The Rise of the Network Society, Blackwell, Oxford.

Drucker P.F. (1992) The Age of Discontinuity, Harper \& Row, New York.

Feller J., Fitzgerald B., Hissam S.A. \& Lakhnani K.R. (2005) Perspectives on Free and Open Source Software, MIT Press, Cambridge, Mass.

Foray D. (2004) The Economics of Knowledge, MIT Press, Cambridge, Mass.

Ghosh R.A., Ruediger G., Krieger B. \& Robles G. (2002) Free/Libre and Open Source Software: survey and study, International Institute of Infonomics, University of Maastricht, Maastricht.

Guellec D. (1999) Economie de l'Innovation, La Découverte, Paris.

Kendrick J.W. (1994) Total capital and economic growth, Atlantic Economic Journal 22 (1).

Kollock P. \& Smith M. (eds) (1999) Communities in Cyberspace, Routledge Press, London.

Kuhn T.S. (1970) The Structure of Scientific Revolutions, University of Chicago Press, Chicago.

Latour B. (1987) Science in Action, Harvard University Press, Cambridge, Mass.

Lazega E. (1998) Réseaux Sociaux et Structures Relationnelles, PUF, Paris.

Malinowski B. (1922) Argonauts of the Western Pacific, John Hawkins, New York.

Mauss M. (1924) Essai sur le don, Année Sociologique 1923-1924, Paris.

McGinn R.E. (1991) Science, Technology and Society, Prentice Hall, New Jersey.

Memmi D. (2006) The nature of virtual communities, AI \& Society 20 (3): 288-300. 
Memmi D. (2014) Information overload and virtual institutions, AI and Society 29 (1): 75-83.

Miller S. (2012) Social institutions, The Stanford Encyclopedia of Philosophy, Edward N. Zalta (ed.), <http://plato.stanford.edu/archives/fall2012/entries/social-institutions/>

Mintzberg H. (1979) The Structuring of Organizations, Prentice Hall, New Jersey.

Orman L.V. (2010) Virtual institutions, Johnson School Research Paper Series \#26-2010, Cornell University, Ithaca, NY.

Rheingold H. (2000) The Virtual Community, MIT Press, Cambridge, Mass.

Scott R. (2001) Institutions and Organisations, Sage, London.

Tönnies F. (1963) Gemeinschaft und Gesellschaft, Wissenschaftliche Buchgesellschaft, Darmstad.

Veltz P. (2000) Le Nouveau Monde Industriel, Gallimard, Paris.

Von Hippel E. (2005) Democratizing Innovation, MIT Press, Cambridge, Mass.

Wasserman S., Faust K. (1994) Social Network Analysis, Cambridge University Press, Cambridge.

Weber M. (1956) Wirtschaft und Gesellschaft, Mohr, Tübingen.

Wellman B. (ed) (1999) Networks in the Global Village, Westview Press, Boulder, CO.

Wikipedia (2013) http://www.wikepedia.org/

W3C (2013) http://www.w3.org/ 